Łukasz M. Plęs

\title{
Der VRP-Neusprech im Spiegel Der Aufzeichnungen VON Michą GŁOWIŃski
}

Der Neusprech als solcher war eine typische Erscheinung der totalitären Diktaturen des zwanzigsten Jahrhunderts. Der Begriff des Neusprechs (engl. Newspeak) stammt aus George Orwells Roman 1984. ${ }^{1}$ Er hat keine strenge linguistische Definition, wird in diversen Bedeutungen verwendet, worauf im Folgenden noch Bezug genommen wird, weist oft eher einen illustrativen Charakter auf. Nach Markowski (2012: 87) stützt sich der Neusprech auf die sprachliche Manipulation, daher sind zur dessen Schilderung auch Manipulationsmechanismen zu analysieren. Markowski gibt auch eine vage Definition des Neusprechs an, die für die Bedürfnisse des vorliegenden Artikels angenommen werden kann:

Die Sprache der Staatsgewalt und der von ihr kontrollierten Verbreitungsmedien in Staaten mit totalitären Systemen, die der breit gefassten Propaganda dient und zur arbiträren Manipulierung von gesellschaftlichen Stimmungen und Verhaltensweisen genutzt wird, aber auch darauf abzielt, die der ganzen Gesellschaft von der Staatsgewalt aufgezwungene Denkweise entsprechend zu gestalten (Markowski 2012: 87, Übers. Ł.M.P.).

Der Neusprech bezieht sich standardmäßig auf die offizielle und propagandistische Sprache der sozialistischen Länder, beziehungsweise der Länder der Volksdemokratie. Der Aufbau des Sozialismus verlief in diesen Ländern nach sowjetischem Vorbild unter ähnlichen Umständen, was sich zwangsläufig in der Sprache (hier in Bezug auf das DDR-Deutsch) manifestierte:

\footnotetext{
${ }^{1}$ Im Roman heißt es: „Neusprech war die Amtssprache Ozeaniens und entworfen worden, um die ideologischen Anforderungen des Engsoz, oder englischen Sozialismus, zu erfüllen. [...] Man erwartete, dass Neusprech etwa bis zum Jahr 2050 Altsprech schließlich verdrängt haben würde. Inzwischen gewann es ständig an Boden, weil alle Parteimitglieder dazu tendierten, im täglichen Sprachgebrauch immer mehr Neusprechwörter und grammatikalische Konstruktionen zu verwenden“ (Orwell 2002: 371).
} 
Der Partei-Apparat beherrschte und bestimmte alles - also auch die offizielle Sprache. Wo ein Genosse ist, ist die Partei, und wo drei davon waren, bildeten sie eine Parteigruppe unter einem Gruppenorganisator, lasen das Parteiprogram, nahmen am Parteilehrjahr teil und wiesen dem Parteisekretär, mindestens jedoch einmal monatlich dem Kassierer, ihr Parteidokument vor (Eik 2010: 32-33).

Im Laufe der vertrackten Umstände, die aus dem Angriff des Dritten Reiches und der Sowjetunion auf Polen 1939 sowie dem Krieg zwischen den bis 1941 verbündeten Diktaturen und dem Siegeszug der Roten Armee resultierten, wurde Polen in den Jahren 1944-1948 ein kommunistisches System in der sowjetischen Ausprägung aufgezwungen. Norman Davies schrieb dazu:

Angesichts ihrer fest begründeten Traditionen und Loyalitäten ist nicht vorstellbar, dass die Polen aus eigenem Antrieb ein kommunistisches Regime hervorgebracht hätten. Es gab kaum genügend einheimische polnische Kommunisten, um eine Fabrik zu leiten, geschweige denn ein Land mit rund dreiBig Millionen Einwohnern (Davies 2006: 3).

Um den Aufbau des Sozialismus zu verwirklichen, setzte man offensichtlich auf Zwangsherrschaft, Terror und nicht zuletzt Propaganda. Die Tristesse der ersten Jahre Polens der Nachkriegszeit, des Stalinismus, wird von Davies folgendermaßen geschildert:

Jeder Kontakt mit der Außenwelt wurde umgehend angeprangert, und es entstand eine allgemeine Atmosphäre, in der politische Prozesse als etwas normales wirkten und harmlose Bürger nach Gutdünken als ausländische Spione verurteilt werden konnten. Man ermutigte die Leute, in Gemeinschaftswohnungen zu leben und kollektiv zu denken. [...] Helden der Arbeit, sogenannte Stachanowarbeiter, wurden verherrlicht und den Massen zur Nachahmung empfohlen. Man förderte den Konformismus in der Bekleidung und im Denken. Eine bestimmte Form von Größenwahn breitete sich aus. [...] Die Arbeiter wurden zu Sklaven ihrer ständig steigenden Arbeitsnormen. In der Kunst galt einzig die Doktrin des Sozialistischen Realismus, Romane feierten Traktorfahrer, Gemälde zeigten Betonfabriken. [...] Lobhudeleien auf die Sowjetunion waren ein Muss. Überall entstanden Stalinstatuen. Kattowitz wurde in »Stalinogród « umbenannt. Man versuchte, die polnische Sprache umzumodeln und die russische Anrede in der zweiten Person Plural, per Wy einzuführen, an Stelle der im Polnischen üblichen Anrede in der dritten Person Singular, per Pan oder Pani (Davies 2006: 8). 
Die polnische Variante des Neusprechs funktionierte von den 50er Jahren bis zum Ausgang der 80er Jahre des vorigen Jahrhunderts (vgl. Zdunkiewicz-Jedynak 2008: 149). Wie bereits erwähnt, bildete sich der Neusprech in allen den Sozialismus aufbauenden Ländern heraus und wies dort jedenfalls sowohl gemeinsame, als auch einzelsprachlich spezifische Charakterzüge auf. Der vorliegende Beitrag setzt sich zum Ziel, einen Einblick in die wesentlichsten Charakteristika des Neusprechs der Volksrepublik Polen zu vermitteln und am Beispiel ausgewählter Aufzeichnungen von Michał Głowiński zu schildern. Sie umfassen eine Zeitspanne zwischen Mitte 60er Jahre bis zum Ausklang des volksdemokratischen Polens. Zunächst für die Schublade geschrieben, wurden sie aus offensichtlichen Gründen erst nach der Wende in einigen Bänden offiziell veröffentlicht. Diese Notizen, die die Propagandasprache unter besonderer Berücksichtigung der Tageszeitung Trybuna Ludu einer scharfen Kritik unterzogen, stellen ein Unikum dar, weil sie auf dem Laufenden entstanden und demzufolge von historischer Bedeutsamkeit sind. Głowińskis Erwägungen beziehen sich ausschließlich auf den Neusprech in der polnischen Abwandlung und können somit nur unter bestimmten Vorbehalten hinsichtlich des Neusprechs in den in anderen Sprachen bekannten Varianten Anwendung finden.

Głowiński stellte sich die Frage, wie man den Sachverhalt der politischen VRP-Sprache hätte bezeichnen können. Im Hinblick auf den Zweck, den sie verfolgte, war sie Propagandasprache. Hinsichtlich der institutionellen Bezugnahme konnte man sie offizielle bzw. Parteisprache, oder - wegen ihrer ideologischen Verknüpftheit - kommunistische Sprache nennen ${ }^{2}$. Da die erwähnten Bezeichnungen den Nachteil haben, dass sie sich jeweils lediglich auf eine Dimension des Phänomens beziehen, entschied sich der Autor für den relativ neutralen, von Orwell übernommenen Termin nowomowa (Neusprech), der erlaubte, das Neue an dieser Sprache im Vergleich mit der klassischen Sprache hervorzuheben, wobei dieses Merkmal nicht umstritten sein konnte und für andere Merkmale keinen Ausschlag gab. Głowiński war sich der Evolution bewusst, der der Neusprech im Verlauf der Jahrzehnte unterlag (was sich auch in den folgenden Bänden widerspiegelt), konzentrierte sich jedoch auf dessen wesentlichste, invariante Merkmale (vgl. Głowiński 2009: 11).

\footnotetext{
${ }^{2}$ An einer anderen Stelle teilte Głowiński (2009: 40ff.) den Neusprech in drei Arten auf, und zwar in den persuasiv-propagandistischen, bürokratischen und kitschig-ludischen Neusprech, wobei die erste Art dominierend war.
} 
Wie Głowiński bemerkte, konnte an das Problem des Neusprechs zweierlei herangegangen werden. Die erste Herangehensweise zielte auf die Erforschung des Neusprechs an sich, auf dessen sprachliche Beschaffenheit und auf dessen semantische Mechanismen ab. Von der anderen Perspektive betrachtet, sei der Neusprech eine historische, soziologische und psychologische Wissensquelle, wobei der Autor betonte, dass sich sein Interesse auf den ersten Aspekt konzentrierte (siehe Głowiński 2009: 12).

Głowiński fasste die grundlegenden Merkmale des Neusprechs in vier Punkten zusammen:

1. Aufzwingung eines ausdrucksstarken wertenden Zeichens.

Dieses Zeichen führte zu transparenten Polarisierungen und zielte auf dezidierte, unbestrittene Bewertung ab. Oft wurden Bewertungen, die zu dichotomischen Aufteilungen führten, wichtiger als die Bedeutung selbst. In der Konsequenz entstand eine Erscheinung, die Głowiński als „lose Semantik“ bezeichnete. Die Bedeutung wurde der Bewertung untergeordnet. Somit war manchmal nicht mehr wichtig, was das Wort bedeutete, sondern wie es qualifiziert wurde, z. B. dobry/zły [gut/schlecht], nasz/obcy [unser/fremd], postępowy/ wsteczny [fortschrittlich/rückschrittlich]. In diesem Sinne bestand der Neusprech in hohem Maße aus vorinterpretierten Bestandteilen. Vor dem Hintergrund der obigen Ausführungen konnte man den Neusprech als eine einwertige Sprache bezeichnen (siehe Głowiński 2009: 12-13).

2. Synthese von pragmatischen und rituellen Elementen.

Der Neusprech zielte auf starke und direkte Einwirkung ab, war laufenden Bedürfnissen untergeordnet und sollte sich im Prinzip an die Umstände, in denen er funktionierte, und an die Rezipienten anpassen. Einerseits setzte das gewisse Flexibilität voraus, andererseits war der Neusprech von Natur aus konservativ und sah davon ab, dass irgendwelche Bestandteile verbraucht waren und ihre pragmatische Wirkung eingebüßt hatten. Die Pragmatizität des Neusprechs wurde prinzipiell von der Ritualität eingeschränkt. Die Ritualität hing mit der Voraussetzung zusammen, dass in bestimmten Situationen nur eine Ausdrucksweise zulässig war. Das Charakteristische am Neusprech war die Verflechtung dieser beiden kontradiktorischen Elemente, wobei sich im Idealfall maximale Ritualität und volle Pragmatizität überlappten (siehe Głowiński 2009: 13). 
3. Bedeutsamkeit der magischen Funktion.

Die Worte bezogen sich auf die Realität und beschrieben diese nicht so sehr, als vielmehr erschufen sie. Das autoritär Ausgedrückte wurde Wirklichkeit. Eklatante Beispiele hierfür waren Slogans. Formal gesehen waren es indikativische Äußerungen. Das Magische äußerte sich nämlich in der sprachlichen Darstellung der gewünschten Zustände in der Art und Weise, als ob sie existent gewesen wären. Und umgekehrt: Wurde eine Bezeichnung ausgelassen ${ }^{3}$, so wies das auf die Inexistenz des Designats hin. Dies betraf sowohl Appellative, als auch Eigennamen (siehe Głowiński 2009: 13-14).

4. Bedeutsamkeit arbiträrer Entscheidungen.

Erwünschte Bezeichnungen, Formeln und Ausdrücke konnten aufgrund einmaliger Entscheidung von einem Tag auf den anderen von der Parteipropaganda zurückgezogen werden, um dann nach demselben Prinzip wiederzukehren. Die Arbitrarität offenbarte sich auch in der willkürlichen Gestaltung der Bedeutungen. Von diesem Blickwinkel her betrachtet war der Neusprech zweifelsohne eine manipulierte Sprache. Von dieser Tatsache zeugten die Schriften der Zensoren mit Vorgaben, worüber und auf welche Art und Weise man schreiben sollte, worüber man keineswegs schreiben durfte, welche Begriffe und Wendungen anzuwenden waren. Daher stellten diese Dokumente eine gewisse Metasprache des Neusprechs ${ }^{4}$ dar (siehe Głowiński 2009: 14).

Unter den oben ausgeführten grundlegenden Merkmalen des Neusprechs rückte Głowiński das erste in den Vordergrund, und zwar die Einwertigkeit (siehe Głowiński 2009: 14):

Das Wort soll im größeren Ausmaß Werte vermitteln als bedeuten, soll von vorneherein interpretiert werden. Die der Semantik überlegene Axiologie soll die Auswahlmöglichkeiten maximal einschränken, dazu führen, dass jedes Wort oder jede Formel bestimmte Werte implizieren, mit ihnen untrennbar verbunden bleiben. Die Wertung kristallisiert sich nicht im Verlauf einer individuellen Äußerung heraus, sondern soll bereits aus ihren Bestandteilen resultieren, soll ihr Ergebnis sein. Die Folgen waren nicht lange zu erwarten: Es hat sich eine ritualisierte und uniformierte Sprache herausgebildet, die nicht

\footnotetext{
${ }^{3}$ Typisch für den Neusprech waren Tabu-Wörter, z. B. inflacja (Inflation), strajk (Streik), kryzys (Krise), vgl. Zdunkiewicz-Jedynak (2008: 150).

${ }^{4}$ In diesem Zusammenhang war das Informationsmonopol der totalitären Staatsgewalt ausschlaggebend. Konstitutive Eigenschaft des Informationsmonopols war die staatliche präventive Zensur (vgl. Markowski 2012: 88).
} 
von dem gestört werden soll, was individuell und frei von festgelegten Wertungsschemata ist (Głowiński 2009: 34, Übers. Ł.M.P.).

Bei der Betrachtung des Neusprechs zog Głowiński drei Termine - Sprache, Rede und Stil - die in der Linguistik präzise voneinander getrennt sind, in Erwägung. Er wies darauf hin, es wäre nicht ausreichend gewesen, den Neusprech mithilfe lediglich eines von denen zu bestimmen. Man könne ihn jedoch als einen Funktionalstil ${ }^{5}$ bezeichnen. Es sprach dafür vor allem, dass der Neusprech Elemente entwickelt hatte, die einen Stil konstituierten, und zwar einen Komplex von Formen, eine bestimmte Phraseologie, deutliche Präferenzen im Bereich der Wortauswahl. Der Begriff des Stils setzte jedoch situationelle Einschränkungen voraus, was dem Anschein nach beim Neusprech der Fall war, was jedoch auch täuschte, da der Neusprech universelle Ansprüche hatte und versuchte, sich andere Bereiche der Sprache unterzuordnen. Zwar war der Neusprech in der politischen Publizistik geboren, ist aber Muster für das Reden im viel breiteren Umfang geworden und strebte nach der Universalität, versuchte nämlich, die Rolle der Sprache zu übernehmen. Daher tendierte Głowiński, den Neusprech als die Quasi-Sprache zu bezeichnen, und zwar mit der Begründung, der Neusprech habe alle Ebenen der Sprache, von der Intonation bis hin zu Satzbaumustern, jedoch mit besonderer Berücksichtigung des Wortschatzes und der Phraseologie in Besitz genommen (vgl. Głowiński 2009: 15).

Głowiński beschrieb verschiedene Methoden der indirekten Einführung der Wertigkeitsindikatoren im Neusprech. Als die einfachste Methode nannte er die Anhäufung von Adjektiven. Manchmal stellten sie einen impliziten Ausdruck normativer Haltungen, z. B. stuszny (richtig), niestuszny (falsch), prawidtowy (korrekt), dar. ${ }^{6}$ In manchen Formeln hatten Adjektive einen rituellen Charakter, in denen sie zwangsläufig vorhanden waren, z. B. przyjacielska wizyta (freundschaftlicher Besuch) (mehr dazu Głowiński 1993: 19). Hin und wieder war eine lockere Verwendung bemerkbar, vorausgesetzt jedoch, dass die Adjektive eine Bewertungskomponente mitbrachten (siehe Głowiński 2009: 16). Die Adjektive spiel-

${ }^{5}$ Markowski (2012: 87f.) betrachtet den Neusprech als im offiziellen, journalistischen, publizistischen und amtlichen Stil bemerkbare Entartung der Sprache, als Wucherung an anderen Stilen.

${ }^{6}$ Ein interessantes Beispiel war die Verwendung des Wortes kontrowersyjny (kontrovers/umstritten) anstelle eines wertenden Attributs in plus oder in minus als Ausdruck einer Distanzierung (im Sinne: einerseits noch nicht falsch, aber andererseits noch nicht empfehlenswert). 
ten im Neusprech eine noch andere Rolle, und zwar die Änderung der Bedeutung des bezeichneten Wortes, insbesondere dort, wo die Formeln als präzise definierte Termini fungieren sollten, z. B. demokracja ludowa (Volksdemokratie). Vorrangige Bedeutung hatte dabei offensichtlich die Evaluierung (vgl. Głowiński 2009: 17).

Ein wesentliches Merkmal des Neusprechs war dessen Beziehung zur immanenten Axiologie der Sprache, d.h. zur Art und Weise, auf die er von Konnotationen, die mit bestimmten Werten zusammenhingen, Gebrauch machte. Ein wertendes Zeichen wurde einem Wort oder einer Formel unabhängig von den Konnotationen in der Gemeinsprache zugeordnet. Somit konnte ein neutrales Wort vorübergehend eine negative Färbung bekommen $^{7}$ (siehe Głowiński 2009: 17).

Interessanterweise wurden im Neusprech sowohl Wörter mit einem ausdrucksstarken Wertungspotenzial, als auch neutrale oder beinahe neutrale Wörter verwendet. Im ersten Fall, wenn das gegebene Wort nicht immer umgestaltet werden konnte, wurde es gegebenenfalls gemieden oder mit Kommentaren versehen. Ein Beispiel hierfür war das Wort wolność (Freiheit), welches im Neusprech relativ selten vorkam und in manchen Kontexten durch wyzwolenie (Befreiung), vorzugsweise mit den Attributen narodowe (national) oder społeczne (gesellschaftlich) ersetzt wurde8 (vgl. Głowiński 2009: 18). Interessant waren in diesem Kontext die im Prinzip neutralen Wörter, welche im Plural verwendet wurden und dem Rezipienten des im Neusprech verfassten Textes eine negative Stellungnahme aufzuwerfen hatten. Dies betraf z. B. solche Wörter wie koła (Kreise), insbesondere mit dem Adjektiv pewne (bestimmte Kreise) versehen. Ähnlich war es beim Wort sity (Kräfte), welches isoliert im Neusprech eine negative Bedeutung hatte ${ }^{9}$.

\footnotetext{
${ }^{7}$ Als eines der Beispiele hierfür nannte Głowiński die gegen Ende der 60er Jahre beleidigende Bezeichnung encyklopedysta (Enzyklopädist), die im Rahmen der Hetzkampagne gegen die Autoren der Großen Allgemeinen Enzyklopädie (Wielka Encyklopedia Powszechna $P W N$ ) verwendet wurde. Einst in den Verkehr gebracht, existierte die Bezeichnung lebhaft und erweiterte den Bedeutungsumfang über die Autoren der Enzyklopädie hinaus. Solch ein starker Bewertungsfaktor führte dazu, dass die grundlegende Bedeutung des Wortes verschwamm, was Ausdruck der losen Semantik war (siehe Głowiński 2009: 17-18; Głowiński 1991: 36).

${ }^{8}$ Die Verwendung des Wortes wolność war insofern interessant, dass es im Prinzip ohne Adjektive vorkam. Dem Wort konnte keine negative Färbung direkt gegeben werden, weil dies mit dem gesellschaftlichen Bewusstsein allzu unvereinbar gewesen wäre. Daher wurde es nur kommentiert (siehe Głowiński 2009: 18).

${ }^{9}$ Etwa im Sinne sity antysocjalistyczne, antypolskie, antyradzieckie (antisozialistische, antipolnische, antisowjetische Kräfte), wobei jedoch positive Kontexte möglich waren,
} 
Ein Wertungspotenzial hatte im Neusprech darüber hinaus die Wortfolge, die die Hierarchie der Begriffe implizierte. Deshalb konnte die Formulierung partia i rzad (Partei und Regierung) keineswegs umgekehrt verwendet werden (siehe Głowiński 1991: 18). Manchmal hatte die Wortfolge einen ritualen Charakter und diente dazu, der Äußerung eine pathetische Färbung zu verleihen. Daher sagte man beispielsweise pokój mitujący naród radziecki (friedensliebendes sowjetisches Volk) anstatt miłujący pokój, was im Polnischen natürlicher erschien (siehe Głowiński 2009: 21).

Bemerkenswert war auch die im Neusprech präsente Metaphorik. Einer deutlichen Evaluierung unterlagen geläufige Metaphern, lexikalisierte Sprüche, Idiome, die mit einem vordefinierten Wertungsindikator vorkamen. Im Neusprech ließ z. B. das Phrasem woda na mtyn (Wasser auf jmds. Mühlen) keine Auswahl zwischen der positiven und negativen Bedeutung zu und war mit einem anderen Phrasem dać żer (Nährboden für etwas darstellen) sinnverwandt (siehe Głowiński 2009: 19; Głowiński 1991: 137-139). Ein anderer Aspekt war die Institutionalisierung der Tropen und Figuren. Die von Gomułka (dem ersten Sekretär der Polnischen Vereinigten Arbeiterpartei in den Jahren 1956-1970) verwendete, zuvor beiläufige Metapher wrogowie wszelkiej maści (Feinde jeglicher Couleur), wurde beinahe obligatorisch für die Bezeichnung der Feinde. Eine noch andere Perspektive war die Militarisierung der Tropen (siehe Głowiński 2009: 21).

Grundlegender Tropus im Bereich des Neusprechs schien nach Głowiński die Periphrase, die Umschreibung der Begriffe durch kennzeichnende Eigenschaften, zu sein. Die Rolle der Periphrase war einfach zu klären, vorausgesetzt, dass die vorrangige Eigenschaft des Neusprechs als Quasi-Sprache in der Hervorhebung der Bewertung bestand. Die Periphrase gab uneingeschränkte Möglichkeiten in diesem Bereich, da sie von Natur aus eine Interpretation ist. Die Periphrase war auch aus dem Grund für den Neusprech besonders von Vorteil, dass sie zur Stabilisierung, zum Funktionieren in der festgelegten Gestalt tendierte. Periphrasen begünstigen demzufolge das, wonach der Neusprech ununterbrochen strebte, und

z. B. sity pokoju, socjalizmu, postępu (Kräfte des Friedens, des Sozialismus, des Fortschritts) (vgl. Głowiński 1993: 191-192; Głowiński 1991: 46; Głowiński 1991: 106). Ein anderes illustratives Beispiel war das Wort nastrój (Stimmung), das im Plural ausschließlich eine negative Färbung hatte, z. B. nastroje antypolskie (antipolnische Stimmungen) (siehe Głowiński 2009: 19). Andere Beispiele führt Zdunkiewicz-Jedynak (2008: 149) an: knowania (Ränke), konszachty (Machenschaften), ekscesy (Exzesse). 
zwar die Umgestaltung der Sprache in einen Komplex von kanonischen Formeln ${ }^{10}$ (siehe Głowiński 2009: 22).

Eine der wichtigsten Eigenschaften des Neusprechs war überdies die Manipulierung mithilfe von Euphemismen und Hyperbeln. Ihr Gebrauch hing mit dem Kontext zusammen. Die Gebrauchsregel konnte folgendermaßen formuliert werden: Werden eigene Probleme oder besorgniserregende Beschlüsse, insbesondere die der wirtschaftlichen Natur, geschildert, treten Euphemismen auf (vgl. Głowiński 1999: 270-271). Werden dagegen Probleme der Gegner geschildert, so ist eine Anreihung von Hyperbeln zu erwarten. Eigene Probleme beschrieb man als vorübergehende Marktschwierigkeiten (przejściowe trudności rynkowe), fremde Probleme dagegen als wirtschaftliche Krise (kryzys ekonomiczny). Demzufolge gab es „bei uns“ keine Preiserhöhungen (podwyżki cen), sondern Preisänderungen (zmiany cen), oder - noch euphemistischer ausgedrückt - Preiskorrekturen (korekty cen), „bei ihnen“ dagegen hatte man mit dem Preisanstieg (wzrost cen) und mit der Inflation (inflacja) zu tun. Das Prinzip war auch andersherum zu deuten. Eigene Erfolge wurden hyperbolisiert, die Erfolge der Gegenpartei euphemisiert. Die Hyperbolisierung konnte darüber hinaus einen ritualen Charakter haben, wovon der Gebrauch des Adjektivs historyczny, z. B. historyczna wizyta (historischer Besuch), historyczne plenum (historisches Plenum) zeugen konnte (siehe Głowiński 2009: 22-23).

Eine interessante Erscheinung, die sich im Rahmen des Neusprechs herausgebildet hatte, war die äsopische Sprache. Sie kam insbesondere dann zum Vorschein, wenn die staatliche Gewalt der Bevölkerung das kommunizieren wollte, was sie expressis verbis nicht kommunizieren konnte. Die äsopische Sprache wurde vor allem in dramatischen Situationen verwendet. Somit konnten die Vorgänge unvorhersehbare Folgen nach sich ziehen (zajścia pociagnąc moga nieprzewidywalne w konsekwencji skutki). Die Mitteilung war konkludent und der Rezipient sollte zwischen den Zeilen lesen. Die äsopische Sprache kam in Einsatz, wenn über etwas gefährliches informiert werden sollte, ohne mitzuteilen, was in Wirklichkeit geschehen war ${ }^{11}$. Dabei blieb vieles unausgesprochen (siehe Głowiński 2009: 24; Głowiński 1996: 40-41).

\footnotetext{
10 Głowiński unterschied auch unter anderem zwischen etikettierenden, z. B. zapluty karzet reakcji (scheußlicher Zwerg der Reaktion), chorąży pokoju (Träger der Friedensfahne) oder wódz postępowej ludzkości (Führer der fortschrittlichen Menschheit) und euphemisierenden Periphrasen, z. B. okres kultu jednostki (Zeit des Persönlichkeitskults) oder okres błędów i wypaczeń (Zeit der Missbräuche und Missstände) (vgl. Głowiński 2009: 46-50).

11 Über die Proteste der Studenten in Krakau 1977 schrieb man enigmatisch to, co zdarzyto się w Krakowie (das, was in Krakau geschehen ist) (siehe Głowiński 2009: 24 und Głowiński 1993: 69-70).
} 
Ein anderes Merkmal des Neusprechs waren reservierte Wörter. Es handelte sich dabei um Wörter, die berechtigt waren, in einem streng bestimmten Kontext vorzukommen und sich auf eine einzige Entität zu beziehen. Ein solches Wort war das für die Partei reservierte Adjektiv kierowniczy (leitend). Die reservierten Wörter waren Ausdruck der für den Neusprech grundlegenden Tendenz, über bestimmte Sachverhalte nur auf vorgeschriebene Art und Weise zu sprechen (siehe Głowiński 2009: 26).

Im Neusprech wurden Synonyme gemieden. Es ging hier nicht um semantische Präzision, sondern um Vermeidung der Missverständnisse oder Ambiguitäten in der Evaluierung. Ein Beispiel dafür war das Wort powiazania (Verbindungen), welches zwar mehrere sinnverwandte Entsprechungen aufwies, jedoch in der negativ markierten Formel mieć powiazania (Verbindungen haben) keineswegs durch diese ersetzt werden konnte. Manchmal wurden Synonyme künstlich eingeführt, um Bezeichnungen mit unerwünschten Konnotationen zu verdrängen. Daher wurden (Lebensmittel)karten (kartki) durch Warengutscheine (bilety towarowe) ersetzt12 (vgl. Głowiński 2009: 29; Głowiński 1993: 7).

Der Neusprech wurde von der regierenden politischen Macht gebildet und in offiziellen sowie propagandistischen Texten verwendet. Diese Erscheinung durchdrang dann die Amtssprache, sodann die Umgangssprache (vgl. Markowski 2012: 87). Entgegen dem Anschein wies der Neusprech einen eingeschränkten Bestand an lexikalisch-phraseologischen Mitteln auf ${ }^{13}$. Er zeichnete sich durch die Stornierung mancher Wörter aus dem Lexikon, durch die Verwandlung, Entstellung und Gestaltung der Bedeutungen für die Bedürfnisse der „einzig richtigen“ Ideologie, durch die Verwendung von festen, förmlich und inhaltlich homogenen Formeln, kanonischen Texten der Parteisekretäre und kognitiven Schemata aus (vgl. Kamińska-Szmaj 2013: 419). Davon zeugt die im Anhang abgedruckte, imaginäre „Phrasen-Drehmaschine“ für den VRP-Neusprech (siehe Tab. 1), die noch in den 80er Jahren kursierte, wobei die in Spalten und Zeilen verteilten Bestandteile beliebig kombinierbar waren. Etwas ähnliches hat auch der aus der DDR geflüchtete SED-Funktionär Franz Loeser

12 Die Manipulation bestand hier darin, dass Konnotationen gemieden wurden, die für die Staatsgewalt ungünstig waren. Die Lebensmittelkarten wurden nämlich mit der Besatzungszeit, mit Unterernährung assoziiert (vgl. Głowiński 1993: 7). Den Arbeiteraufstand vom Dezember 1970 bezeichnete man als zajścia (Zwischenfälle), um andere Bezeichnungen, z. B. bunt (Meuterei), rewolta (Revolte), zu verdrängen (vgl. Głowiński 2009: 29).

${ }^{13}$ Der Neusprech sah von der Lexik ab, die sich auf Ethik, Moral und Religion bezog, und machte stattdessen von etikettierenden Bezeichnungen Gebrauch, z. B. klika (Clique), twardogtowi (Betonköpfe) (vgl. Zdunkiewicz-Jedynak 2008: 151). 
entwickelt (siehe Tab. 2 im Anhang). Andererseits bot der Neusprech, wie Schneider bemerkt, „subtil unterscheidende Vokabeln für alles an, was die Partei zu denken erlaubt[e]“ (Schneider 1992: 149).

\section{Literaturverzeichnis}

Davies, Norman (2006): Im Herzen Europas. Geschichte Polens (aus dem Englischen von Friedrich Griese). München.

Eik, Jan (2010): DDR-Deutsch. Eine entschwundene Sprache. Berlin.

Głowiński, Michał (1991): Marcowe gadanie. Komentarze do słów 1966-1971. Warszawa.

Głowiński, Michał (1993): Peereliada. Komentarze do słów 1976-1981. Warszawa.

Głowiński, Michał (1996): Mowa w stanie oblężenia. 1982-1985. Warszawa.

Głowiński, Michał (1999): Końcówka (czerwiec 1985-styczeń 1989). Kraków.

Głowiński, Michał (2009): Nowomowa i ciągi dalsze. Szkice dawne i nowe. Kraków.

Kamińska-Szmaj, Irena (2013): Język propagandy politycznej okresu PRL. In: Malinowska, Ewa et al. (Hg.): Style współczesnej polszczyzny. Przewodnik po stylistyce polskiej. Kraków, S. 419-422.

Markowski, Andrzej (2012): Kultura języka polskiego. Teoria. Zagadnienia leksykalne. Warszawa.

Orwell, George (2002): 1984. Roman (aus dem Englischen von Michael Walter). München.

Panskus, Hartmut (Hg.) (1986): Zweimal Deutschland. Fakten und Funde zur geteilten Lage der Nation. München.

Schneider, Wolf (1992): Wörter machen Leute. Magie und Macht der Sprache. München.

Zdunkiewicz-Jedynak, Dorota (2008): Wykłady ze stylistyki. Warszawa.

\section{Nowomowa PRL w świetle zapisków Michała Głowińskiego}

Abstrakt: Niniejszy artykuł podejmuje temat nowomowy używanej w Polskiej Rzeczypospolitej Ludowej ze szczególnym uwzględnieniem komentarzy Michała Głowińskiego. Komentarze powstawały od połowy lat sześćdziesiątych do schyłku PRL i opierały się przede wszystkim na lekturze „Trybuny Ludu”. Głowiński poddaje ostrej krytyce i wnikliwej analizie język propagandy władzy państwowej, obnażając mechanizmy manipulacji. Zapiski zostały oficjalnie wydane w kilku tomach dopiero w latach dziewięćdziesiątych, mają jednakże unikatową wartość ze względu na fakt, że powstawały na bieżąco, w okresie, kiedy nowomowa PRL funkcjonowała.

Słowa kluczowe: nowomowa, Polska Rzeczpospolita Ludowa, socjalizm, propaganda, manipulacja językowa. 


\title{
The Newspeak of the Polish People's Republic in the Mirror of the Comments by Michał Głowiński
}

\begin{abstract}
This article deals with the issue of the newspeak as it was used in the Polish People's Republic, with special regard to the remarks by Michał Głowiński. The comments were created from the mid-sixties to the decline of the Polish People's Republic and based on the reading of the daily "Trybuna Ludu" ("People's Tribune"). Głowiński provides an in-depth analysis and levels harsh criticism at the propaganda language of the state authorities, revealing the mechanisms of manipulation. The notes were officially published in a few volumes not until the nineties, however they are unique due to the fact that they came into being systematically at the time the newspeak of the Polish People's Republic was used.
\end{abstract}

Key words: newspeak, Polish People's Republic, socialism, propaganda, linguistic manipulation. 


\section{ANHANG}

Tab. 1

Die „Phrasen-Drehmaschine“ für den VRP-Neusprech

\begin{tabular}{|c|c|c|c|}
\hline $\begin{array}{l}\text { Koleżanki } \\
\text { i koledzy }\end{array}$ & $\begin{array}{l}\text { realizacja nakre- } \\
\text { ślonych zadań } \\
\text { programowych }\end{array}$ & $\begin{array}{l}\text { zmusza nas do } \\
\text { przeanalizowania }\end{array}$ & $\begin{array}{l}\text { istniejących warun- } \\
\text { ków administracyj- } \\
\text { no-finansowych }\end{array}$ \\
\hline $\mathrm{Z}$ drugiej strony & $\begin{array}{l}\text { zakres i miejsce } \\
\text { szkolenia kadr }\end{array}$ & $\begin{array}{l}\text { spełnia istotną rolę } \\
\text { w kształtowaniu }\end{array}$ & $\begin{array}{l}\text { dalszych kierun- } \\
\text { ków rozwoju }\end{array}$ \\
\hline Podobnie & $\begin{array}{l}\text { stały wzrost ilości } \\
\text { i zakres naszej } \\
\text { aktywności }\end{array}$ & $\begin{array}{l}\text { wymaga sprecy- } \\
\text { zowania i okre- } \\
\text { ślenia }\end{array}$ & $\begin{array}{l}\text { systemu po- } \\
\text { wszechnego } \\
\text { uczestnictwa }\end{array}$ \\
\hline $\begin{array}{l}\text { Nie zapominajmy } \\
\text { jednak, że }\end{array}$ & $\begin{array}{l}\text { aktualna struktu- } \\
\text { ra organizacji }\end{array}$ & $\begin{array}{l}\text { pomaga w przy- } \\
\text { gotowaniu i reali- } \\
\text { zacji }\end{array}$ & $\begin{array}{l}\text { postaw uczest- } \\
\text { ników zadań } \\
\text { stawianych przez } \\
\text { organizację }\end{array}$ \\
\hline W ten sposób & $\begin{array}{l}\text { nowy model dzia- } \\
\text { łalności organiza- } \\
\text { cyjnej }\end{array}$ & $\begin{array}{l}\text { zabezpiecza udział } \\
\text { szerokiej grupie } \\
\text { w kształtowaniu }\end{array}$ & $\begin{array}{l}\text { nowych propo- } \\
\text { zycji }\end{array}$ \\
\hline $\begin{array}{l}\text { Praktyka dnia } \\
\text { codziennego } \\
\text { dowodzi, że }\end{array}$ & $\begin{array}{l}\text { dalszy rozwój } \\
\text { różnych form } \\
\text { działalności }\end{array}$ & $\begin{array}{l}\text { spełnia ważne } \\
\text { zadania w wypra- } \\
\text { cowaniu }\end{array}$ & $\begin{array}{l}\text { kierunków postę- } \\
\text { powego wycho- } \\
\text { wania }\end{array}$ \\
\hline $\begin{array}{l}\text { Wagi i znaczenia } \\
\text { tych problemów } \\
\text { nie trzeba udo- } \\
\text { wadniać, ponie- } \\
\text { waż }\end{array}$ & $\begin{array}{l}\text { stałe zabezpiecze- } \\
\text { nie informacyjno- } \\
\text {-propagandowe } \\
\text { naszej działal- } \\
\text { ności }\end{array}$ & $\begin{array}{l}\text { umożliwia } \\
\text { w większym stop- } \\
\text { niu tworzenie }\end{array}$ & $\begin{array}{l}\text { systemu szkolenia } \\
\text { kadry odpowiada- } \\
\text { jącego potrzebom }\end{array}$ \\
\hline $\begin{array}{l}\text { Różnorakie } \\
\text { i bogate doświad- } \\
\text { czenia }\end{array}$ & $\begin{array}{l}\text { wzmacnianie } \\
\text { i rozwijanie struk- } \\
\text { tur }\end{array}$ & $\begin{array}{l}\text { powoduje doce- } \\
\text { nienie wagi }\end{array}$ & $\begin{array}{l}\text { odpowiednich } \\
\text { warunków akty- } \\
\text { wizacji }\end{array}$ \\
\hline $\begin{array}{l}\text { Troska organiza- } \\
\text { cji, a szczególnie }\end{array}$ & $\begin{array}{l}\text { konsultacja z sze- } \\
\text { rokim aktywem }\end{array}$ & $\begin{array}{l}\text { przedstawia } \\
\text { interesującą próbę } \\
\text { sprawdzenia }\end{array}$ & modelu rozwoju \\
\hline $\begin{array}{l}\text { Wyższe założenia } \\
\text { ideowe, a także }\end{array}$ & $\begin{array}{l}\text { rozpoczęcie } \\
\text { powszechnej akcji } \\
\text { kształtowania } \\
\text { postaw }\end{array}$ & $\begin{array}{l}\text { pociąga za sobą } \\
\text { proces wdrażania } \\
\text { i unowocześnia- } \\
\text { nia }\end{array}$ & $\begin{array}{l}\text { form oddziały- } \\
\text { wania }\end{array}$ \\
\hline
\end{tabular}

Quelle: nach Zdunkiewicz-Jedynak 2008: 154. 
Tab. 2

Die „Phrasen-Drehmaschine“ für den DDR-Neusprech

\begin{tabular}{|l|l|l|}
\hline fortschrittliche & Bündnis- & -bewegung \\
\hline gesellschaftliche & Entwicklungs- & -beziehung \\
\hline ideologische & Erziehungs- & -bildung \\
\hline imperialistische & Friedens- & -einstellung \\
\hline marxistisch-leninistische & Gemeinschafts- & -frage \\
\hline parteiliche & Integrations- & -initiative \\
\hline schöpferische & Klassen- & -konzeption \\
\hline soziale & Persönlichkeits- & -politik \\
\hline sozialistische & Planungs- & -problematik \\
\hline weltanschauliche & Solidaritäts- & -tendenz \\
\hline
\end{tabular}

Quelle: nach Panskus 1986: 155. 